\title{
Synthesis and In Vitro Antigungal Properties of 4-Aryl-4-N- arylamine-1-butenes and Related Compounds
}

\author{
V. Kouznetsov ${ }^{1}$, J. Urbina ${ }^{1}$, A. Palma ${ }^{1}$, S. López ${ }^{2}$, C. Devia ${ }^{3}$, R. Enriz ${ }^{3}$ and S. Zacchino ${ }^{2}$ \\ ${ }^{1}$ Quím. Org. Fina, Fac. Quím., Univ. Ind. de Santander, A.A. 678, Bucaramanga, Colombia \\ ${ }^{2}$ Farmacognosia, Fac.Cs. Bioq. y Farm., Univ.Nac de Rosario, Suipacha 531, (2000)-Rosario \\ E-mail:szaabgil@citynet.net.ar \\ ${ }^{3}$ Quím.Gral, Fac. de Quím., Bioq. y Farm., Chacabuco y Pedernera, (5700)-San Luis, Argentina
}

\begin{abstract}
A new series of 4-aryl and 4-alkyl-4-N-arylamine-1-butenes (homoallylamines) were synthesized and some of them transformed to 4-aryl or alkylquinolines. All of them showed strong antifungal activities against human pathogenic fungi in vitro, being Epidermophyton floccosum the most susceptible species.
\end{abstract}

\section{Introduction}

As part of our project devoted to the search for antifungal agents [1-3], we synthesized a series of new 4-aryl- or 4-alkyl-N-arylamine-1-butenes and transformed some of them into biologically important 2-substituted 4-methyl-tetrahydroquinolines and quinolines [4]. We evaluated them for antifungal properties with agar dilution assays and studied their structure-activities relationships (SAR).

\section{Experimental}

Chemistry. Homoallylamines 12-22 were prepared via the addition of Grignard reagent to aldimines 1-11. Electrophilic cyclization of two of them, compounds 12 and 13 under acidic conditions, led to tetrahydroquinolines 23 and 24, which were oxidised to quinolines 25 and 26 with DDQ (Scheme 1)

Microorganisms. We used standardized human pathogenic fungi from CEREMIC or ATCC. at concentrations up to $50 \mu \mathrm{g} / \mathrm{mL}[1,2]$.

Antifungal evaluation. The dilution agar method was used according with reported procedures [1,2].

\section{Results and Discussion}

All compounds tested showed antifungal properties against dermatophytes $(3.12<\mathrm{MIC}<50 \mu \mathrm{g} / \mathrm{mL})$, in particular against Epidermophyton floccosum, similar to those obtained with Amphotericin or Ketoconazole (Table 1). Substituents on benzene rings A or B increased four times the activity respective the non-substituted analogs. The change of an OMe from position 4 to 2 in rings A or B increased the activity twice. 
Scheme 1

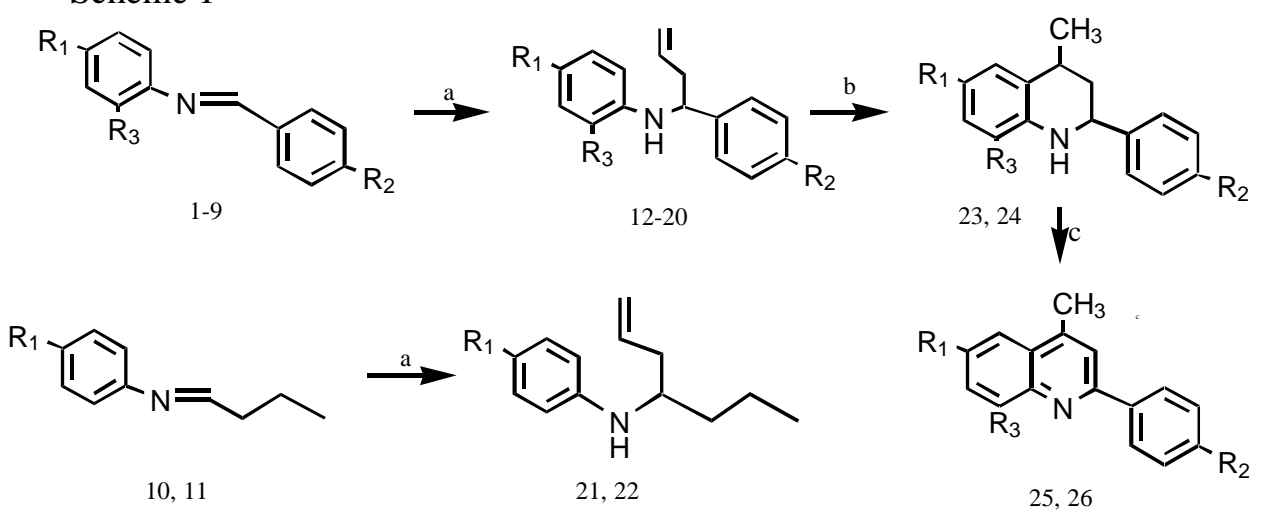

a)Allyl bromide $+\mathrm{Mg} / \mathrm{Et}_{2} \mathrm{O}, 1 \mathrm{O}^{\circ} \mathrm{C}$; b) $\mathrm{H}_{2} \mathrm{SO}_{4} 75 \% \mathrm{~W} / \mathrm{V}$; c) DDQ/Bz,

Table I. MIC values $(\mu \mathrm{g} / \mathrm{mL})$ of homoallylamines, tetrahydroquinolines and quinolines acting against dermatophytes.

\begin{tabular}{|c|c|c|c|c|c|c|c|c|c|}
\hline & C & & D & & E & \multicolumn{4}{|c|}{$\mathrm{F}$} \\
\hline Compd & $\begin{array}{c}\text { Typ } \\
\mathrm{e}\end{array}$ & $\mathrm{R}_{1}$ & $\mathrm{R}_{2}$ & $\mathrm{R}_{3}$ & $\begin{array}{l}M . \\
c .{ }^{a}\end{array}$ & $\underset{b}{M . g}$ & $\begin{array}{c}T . \\
m \cdot{ }^{c}\end{array}$ & T. $r^{d}$ & $E . f^{e}$ \\
\hline 12 & $\mathrm{C}$ & $\mathrm{H}$ & $\mathrm{H}$ & $\mathrm{H}$ & 30 & 30 & 30 & 30 & 12.5 \\
\hline 13 & $\mathrm{C}$ & $\mathrm{CH}_{3}$ & $\mathrm{H}$ & $\mathrm{H}$ & 30 & $>50$ & $>50$ & $>50$ & 3.12 \\
\hline 14 & $\mathrm{C}$ & $\mathrm{OCH}_{3}$ & $\mathrm{H}$ & $\mathrm{H}$ & 30 & $>50$ & 30 & 30 & 3.12 \\
\hline 15 & $\mathrm{C}$ & $\mathrm{F}$ & $\mathrm{H}$ & $\mathrm{H}$ & $>50$ & $>50$ & $>50$ & $>50$ & 30 \\
\hline 16 & $\mathrm{C}$ & $\mathrm{Cl}$ & $\mathrm{H}$ & $\mathrm{H}$ & $>50$ & $>50$ & $>50$ & $>50$ & 30 \\
\hline 17 & C & $\mathrm{Br}$ & $\mathrm{H}$ & $\mathrm{H}$ & $>50$ & $>50$ & $>50$ & $>50$ & 30 \\
\hline 18 & $\mathrm{C}$ & $\mathrm{H}$ & $\mathrm{OCH}_{3}$ & $\mathrm{H}$ & 30 & $>50$ & $>50$ & $>50$ & 3.12 \\
\hline 19 & $\mathrm{C}$ & $\mathrm{Cl}$ & $\mathrm{N}\left(\mathrm{CH}_{3}\right)_{2}$ & $\mathrm{H}$ & $>50$ & $>50$ & $>50$ & $>50$ & $>50$ \\
\hline 20 & $\mathrm{C}$ & $\mathrm{H}$ & $\mathrm{H}$ & $\mathrm{CH}_{3}$ & 30 & $>50$ & $>50$ & $>50$ & 3.12 \\
\hline 21 & D & $\mathrm{H}$ & - & - & $>50$ & $>50$ & $>50$ & $>50$ & $>50$ \\
\hline 22 & $\mathrm{D}$ & $\mathrm{CH}_{3}$ & - & - & $>50$ & $>50$ & $>50$ & $>50$ & $>50$ \\
\hline 23 & $\mathrm{E}$ & $\mathrm{H}$ & $\mathrm{H}$ & $\mathrm{H}$ & 50 & 25 & 25 & 25 & 12.5 \\
\hline 24 & $\mathrm{E}$ & $\mathrm{CH}_{3}$ & $\mathrm{H}$ & $\mathrm{H}$ & 50 & 25 & 25 & 25 & 12.5 \\
\hline 25 & $\mathrm{~F}$ & $\mathrm{H}$ & $\mathrm{H}$ & $\mathrm{H}$ & 25 & 12.5 & 12.5 & 25 & 12.5 \\
\hline 26 & $\mathrm{~F}$ & $\mathrm{CH}_{3}$ & $\mathrm{H}$ & $\mathrm{H}$ & 0.75 & 12.5 & 25 & 12.5 & 12.5 \\
\hline Amp. & & & & & $>50$ & 6.25 & 6.25 & 25 & 0.3 \\
\hline Ket. $^{g}$ & & & & & 15 & 6.25 & 12.5 & 15 & 25 \\
\hline
\end{tabular}

${ }^{\mathrm{a}}$ Microsporum canis $\mathrm{C} 112 .{ }^{\mathrm{b}}$ Microsporum gypseum C 115 . $^{\mathrm{c}}$ Trichophyton mentagrophytes ATCC 9972. ${ }^{\mathrm{d}}$ Trichophyton rubrum C 113 . ${ }^{\mathrm{e}}$ Epidermophyton floccosum C $114 .{ }^{\mathrm{f}} \mathrm{Amp}=\mathrm{amphotericin} \mathrm{B}$.

${ }^{\mathrm{g}}$ Ket.=ketoconazole. 


\section{References and Notes}

1. Zacchino, S.; Rodríguez, G.; Pezzenati, G.; Orellana, G.; Enriz, D.; González Sierra, M. J. Nat. Prod. 1997, 60, 659-662.

2. Zacchino, S.; Santecchia, C.; López, S.; Gattuso, S.; Muñoz, J.; Cruañes, A.; Vivot, E.; Salinas, A.; Ruiz, R.; Ruiz, S. Phytomedicine 1998, 5, 389-395.

3. Rodríguez, A,; Giannini, F.; Baldoni, H.; Suvire, F.; Zacchino, S.; Sosa, C.; Enriz, R.; Csazar, P.; Czismadia, I. J. of Molecular Structure (TEOCHEM) 1999, 463, 283-303.

4 Kuznetsov, V.; Andreeva, E.; Prostakov, N. Khim. Farm. Zh.1995, 29, 61-62; (1996) Chem. Abst. $124,48.290$. This work is part of: Urbina et al., Inhibitors of the fungal wall. Synthesis of 4-aryl4-N-arylamine-1-butenes and related compounds with inhibitory activities on $\beta(1-3)$ glucan and chitin synthases, Bioorganic \& Med. Chem., in press 